\title{
Reducing repeated chlamydia and gonorrhoea infections
}

\author{
Sonia Raffe, Helen Patrick, Rebecca Murdock, Jozette Power, Gill Dean \\ Brighton and Sussex University Hospitals NHS Trust, Brighton
}

\section{$\Leftrightarrow \quad-\infty$ Introduction}

The role of sexual health services is not only to treat sexually transmitted infections (STIs) but to also reduce repeat infections through appropriate antimicrobials, health education and partner notification. Chlamydia and gonorrhoea are both common bacterial STIs which while curable with appropriate antimicrobials, when untreated can cause significant morbidity. Untreated infection can have an impact on fertility, particularly in the context of repeat infection. The British Association for Sexual Health and HIV (BASHH) provides guidance on the management of both these infection and on the use of partner notification.

\section{Aim}

We reviewed the management of patients with repeated chlamydia and gonorrhoea infections to see if the standards outlined in the BASHH guidelines had been met and to identify any areas for improvement.

\section{Methods}

Patients attending the Sexual Health and Contraception Service (SHAC) with more than one episode of chlamydia and/or gonorrhoea between July 2015 and June 2016 were identified via clinic coding. A retrospective case-not review of their attendances was performed.

\section{Results}

156 patients were identified of which a random sample of $30(20 \%)$ were reviewed. All were male; $70 \%(21)$ were men who have sex with men (MSM), 23\% (7) were heterosexual and 7\% (2) were bisexual. The median age was 29.5 years (range $21-58$ ).

- $77 \%$ (23) had 2 infective episodes in the audit period, 23\% (7) had 3 episodes

- Of the 67 episodes of infection 53\% (36) were gonorrhoea, 39\% (26) were chlamydia and 7\% (5) were both

- All episodes were treated with appropriate antimicrobials

- $40 \%$ (27) were treated the same day, 9\% (6) within 1 week, $24 \%$ (16) between $1-2$ weeks and $22 \%$ between 2-4 weeks

$57 \%(17)$ had a documented risk factor for unsafe sex with $10 \%(3)$ reporting multiple factors

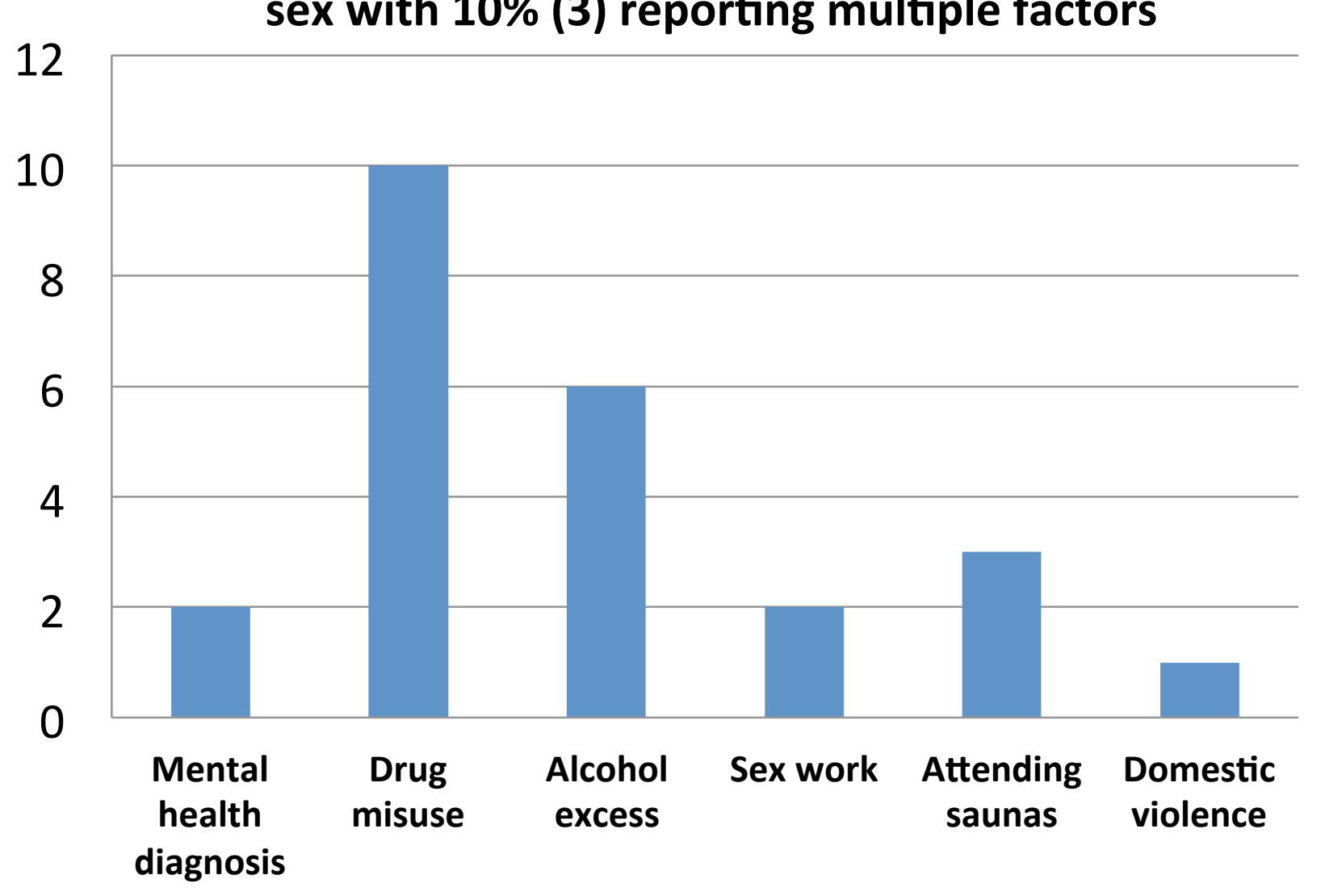

- $30 \%$ (9) were HIV positive, all of whom identified as MSM

- Of this group $78 \%$ (7/9) had undetectable HIV viral loads throughout the audit period, established on antiretroviral therapy (ART)

- $22 \%$ (2/9) were ART naïve with viral loads of $>6000$ and $>300$ copies per $\mathrm{mL}$ respectively

- All HIV negative patients underwent HIV testing at least twice during the audit period

- Two patients (7\%) have since been diagnosed with HIV

\section{Patients reported between 1 and 100} partners in the 6 months prior to review

- Patients reported between 1 and 100 partners in the 6 months prior to review

- Following the 67 infective episodes $73 \%$ (48) saw a health advisor (HA). In the remaining $28 \%$ the most common reason for not seeing a HA was being managed in non-sexual health clinic e.g. PEP clinic, research clinic or a general HIV clinic.

- Partner notification was undertaken in $82 \%$ (55) of episodes although only completed in $52 \%$ (35) due to untraceable partners

\section{Discussion}

- A high proportion of patients with repeat infections had risk factors for unsafe sex. Focusing on addressing these may facilitate a reduction in repeat STIs

- While most patients were able to access a HA for support, referral pathways from non-sexual health clinics needs improving

- Partner notification remains challenging in the context of multiple casual partners and novel strategies such as electronic partner notification should be introduced urgently 